\title{
A hybrid finite element approach to modelling sound radiation from circular and rectangular ducts.
}

\author{
Wenbo Duan \\ School of Engineering and Design, \\ Mechanical Engineering, \\ Brunel University, \\ Uxbridge, Middlesex, UB8 3PH, UK.
}

\author{
Ray Kirby* \\ School of Engineering and Design, \\ Mechanical Engineering, \\ Brunel University, \\ Uxbridge, Middlesex, UB8 3PH, UK. \\ ray.kirby@brunel.ac.uk
}

Running Title: sound radiation from ducts

* Corresponding author. 


\begin{abstract}
A numerical model based on a hybrid finite element method is developed, which seeks to join sound pressure fields in interior and exterior regions. The hybrid method is applied to the analysis of sound radiation from open pipes, or ducts, and uses mode matching to couple a finite element discretisation of the region surrounding the open end of the duct, to wave based modal expansions for adjoining interior and exterior regions. The hybrid method facilitates the analysis of ducts of arbitrary but uniform cross-section, as well the study of conical flanges and here a modal expansion based on spherical harmonics is applied. Predictions are benchmarked against analytic solutions for the limiting cases of flanged and unflanged circular ducts and excellent agreement between the two methods is observed. Predictions are also presented for flanged and unflanged rectangular ducts, and since the hybrid method retains the sparse banded and symmetric matrices of the traditional finite element method, it is shown that predictions can be obtained within an acceptable timeframe even for a three dimensional problem.
\end{abstract}




\section{INTRODUCTION}

Understanding the radiation of sound from ducts or pipes is important in many areas of engineering, for example in the radiation of sound from exhaust systems, musical instruments and HVAC ductwork. It is, therefore, not surprising that the study of radiation from an open pipe, or duct, has received extensive attention in the literature. Here, sound radiation depends both on the characteristics of the sound source, but also on the geometry of the duct and the way in which it is terminated, normally either with or without a flange. This represents a very general problem that involves joining interior and exterior sound fields, which presents a significant computational challenge. Accordingly, the vast majority of publications in the literature have been restricted to the classical axisymmetric problem of sound radiation from a flanged or unflanged circular duct. This article seeks to extend this work to the more general problem, which includes radiation from ducts of arbitrary but uniform cross sections, as well as accommodating a flange that may take on any angle $\alpha$ between the geometrical limits of unflanged $\left(\alpha=0^{\circ}\right)$ and flanged $\left(\alpha=90^{\circ}\right)$. To maintain this general approach a finite element method based on the hybrid method of Kirby ${ }^{1}$ is adopted here. This enables a computationally efficient treatment of the problem, as well as avoiding the use of perfectly matched layers (PMLs) typically found in commercial finite element software. Sound radiation is quantified here in terms of the duct end correction, since this is the traditional method for sound radiation problems of this type, as well as duct transmission loss which is important in the design of exhaust pipes and HVAC ductwork.

Work on the radiation of sound from ducts goes back to Rayleigh ${ }^{2}$, who provides an approximate analysis for the length correction of a circular-sectioned duct fitted with a flange that is assumed to be infinite in length (hereafter all references to a flanged duct assume that 
this flange extends to infinity in all directions). Rayleigh assumed that the axial velocity distribution over the aperture is either constant or a polynomial function of the distance from the centre of the duct. This enabled Rayleigh to obtain a static (zero frequency) end correction coefficient $\delta / a=0.82$, (where $a$ is the radius of the circular duct). Rayleigh also investigated experimentally the radiation from an unflanged duct and here an end correction coefficient of 0.6 was measured. Another classical work is that of Levine and Schwinger ${ }^{3}$ who developed an analytic formulation for the end correction of an unflanged circular duct using the Wiener-Hopf technique. Their solution is restricted to plane wave propagation within the duct and so this model is valid only below the cut-on frequency of the first radial duct mode. The static end correction coefficient calculated by Levine and Schwinger ${ }^{3}$ is 0.6133, which compares well to the value measured by Rayleigh, although Levine and Schwinger's method does not readily lend itself to the analysis of a flanged duct. Several authors later provide curve fitting formulae for Levine and Schwinger's end correction coefficient ${ }^{4-6}$. The Wiener-Hopf technique was also applied by $\mathrm{Ando}^{7}$, and later by Bernard and Denardo ${ }^{8}$, but this time to investigate the effect of wall thickness on the end correction from an unflanged circular duct. This involved increasing the thickness of the pipe wall so that the behaviour of the duct begins to take on the characteristics of a flanged duct. In order to deliver a more efficient analytic approach Homicz and Lordi ${ }^{9}$, and later Joseph and Morfey $^{10}$, demonstrate that the pressure in the acoustic far field of the exterior domain may be related to the modes in an unflanged circular duct by use of an appropriate transfer function. This transfer function was derived using the Wiener-Hopf technique and is presented in a form suitable for practical computations since it has the same mode order dependence as the incident modes, and the exterior domain pressure also has the same circumferential angular dependence as the pressure in the interior. Sinayoko et al. ${ }^{11}$ later added mean flow effects using the same modal based technique. The advantage of this 
technique is that the modal directivity patterns for the radiation field can be observed in a straightforward way when compared to the integral formulae of Levine and Schwinger; however, both techniques are limited to unflanged circular ducts, and the Wiener-Hopf technique cannot be readily extended to a more general problem such as the study of rectangular ducts.

For a flanged circular duct the external pressure field is most widely expressed using a Green's function integral. This method has the advantage that on the surface of the flange the normal derivative of the Green's function is zero, thus the integration of the pressure field in the exterior domain only needs to be carried out over the duct aperture. For example, a Green's function integral is adopted by Zorumski ${ }^{12}$, Norris and Sheng ${ }^{13}$, and Wendoloski et $a l .{ }^{14}$ Here, Norris and Sheng calculate a static end correction coefficient of 0.82159 for a flanged circular duct, whereas Wendoloski et al. propose the range $0.82166<\delta / a<0.82168$. Nomura et al. ${ }^{15}$ adopt an alternative method based on the use of Weber-Schafheilin type integrals which may then be expressed in the form of Jacobi's polynomial expansion; this delivers a static end correction coefficient of 0.8217 . One disadvantage of these models is that they do not deliver a closed form solution for the radiated sound field, and so the speed and accuracy of the solution relies on the rates of convergence after truncating a set of infinite sums. Accordingly, Green's function based methods are often computationally slow, especially when modelling sound radiation from ductwork. To address this, Bom and Park ${ }^{16}$, and later Amir et al. ${ }^{17}$, present a simpler form for the pressure field in the exterior domain by assuming only a single forward travelling mode, which is expressed in the form of a Fourier transform; this allows for simplification of the final algorithms but still requires the duct geometry to be axisymmetric. This approach also requires the truncation of an infinite number of equations, although Amir et al. show that the convergence of the axial velocity 
field over the aperture of a flanged circular duct can be achieved much more quickly by adding an extra axial velocity continuity interface.

It is evident that analytic solutions present many computational challenges and there are, inevitably, limitations on the duct geometry one can accommodate using this approach. Accordingly, numerical methods are necessary for studying more general duct radiation problems. For example, Silva and Scavone ${ }^{18}$ and Silva et al. ${ }^{19}$ use the lattice Boltzmann method to evaluate the far-field normal mode radiation pattern from an unflanged circular duct. They ignore circumferential pressure variations so the model is valid only for axisymmetric modes, but this method is capable of capturing the effects of mean flow. However, in order to apply the far field boundary condition the model requires a very large fluid domain, and even then this can only approximate the true anechoic condition. The boundary element method (BEM) has also been applied by several authors and in principle this method is capable of examining ducts of arbitrary cross-section, although to date only radiation from ducts with a circular cross-section has been examined ${ }^{5,20,21}$. For example, Dalmont $e t a l^{5}$ examined unflanged circular ducts, where they found it necessary to assign a finite length duct in place of the (nominally) infinite duct when modelling the duct exterior, and so assume that the effect of this on the computed end correction is negligible. Dalmont $e t$ al. do, however, note that their BEM approach gives inaccurate results for values of Helmholtz number less than 0.18 (based on duct radius); this is probably due to the BEM finding it difficult to resolve low values of acoustic pressure in the exterior sound field. To overcome this, Dalmont et al. use the finite difference method (FDM) to model low values of Helmholtz number, but this approach cannot properly model the exterior domain and so the two models have to be linked together to deliver predictions over a wide range of Helmholtz number. Clearly, this approach is far from satisfactory, and a further well-known disadvantage of the BEM is that it delivers a very full final system matrix because of the 
inherent global connectivity of the method. This means that in order to maintain acceptable solution times one must normally economise on the total number of degrees of freedom (DOF). In fact, it is noticeable that all of the numerical calculations reported using the BEM adopt relatively modest duct dimensions, whereby a duct length of three to four times the radius is the norm, although some results are available for up to ten times the radius. Furthermore, BEM predictions are currently limited to circular (axisymmetric) geometries. One obvious alternative to the BEM is to use an infinite element based method to model the exterior sound field, since this method generates a simple approximation of the radiated sound pressure by use of a sum of functions based on the leading terms of lower-order

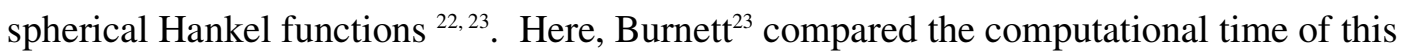
method with the BEM and reported that the infinite element method is two or three orders of magnitude faster than the BEM for the same accuracy. However, infinite elements are not well suited to modelling ducts with a flange, since the method requires an artificial boundary that encloses all of the structure; moreover, Burnett models radiation from a vibrating surface and so it is not immediately obvious how this can be efficiently joined to an interior region.

The standard FEM approach, such as the one presented by Lau and Tang ${ }^{24}$, and that typically found in commercial packages, represents an exterior domain by meshing a region of this domain and then adding absorptive "walls", or PMLs, to form an artificial boundary on the outer limits of this domain. This method attempts to damp down progressive acoustic waves and so numerically enforces a quasi-anechoic termination condition ${ }^{25}$. For the problem considered here this would involve assigning a PML to both the interior and exterior domains. Furthermore, to obtain a good approximation of a non-reflecting boundary in the exterior domain it appears likely that this boundary would need to be placed a significant distance from the duct. This is potentially very computationally expensive, as the number of degrees of freedom in the exterior domain can quickly become prohibitive, especially at 
higher frequencies. Thus, the standard FEM approach is perhaps only suited to modelling low frequency sound radiation problems and/or axisymmetric geometries such as flanged circular ducts of modest dimensions where symmetry can lower the size of the problem. Furthermore, PMLs can only provide an approximation of a true anechoic boundary condition and it is preferable to avoid such approximations where possible. Accordingly, this article seeks to remove the need for PMLs as well as adopting a more efficient modelling approach that economises on the number of degrees of freedom required for a particular problem. Here, Astley ${ }^{26}$ demonstrates that a potentially more efficient approach would be to use a modal expansion for the exterior sound field, as this removes the need for a PML. Astley used Hankel functions to generate an analytic modal expansion for an exterior sound field, and coupled this to a radiating surface using a mode matching method, although he did not consider joining interior and exterior sound fields or conical flanges. This method does, however, demonstrate that it is possible to develop a more efficient FE based approach, and so this article draws on the principles behind this method and presents a FE based technique that seeks only to discretise a small region near the open mouth of a radiating duct. This FE discretisation is then used to join the interior and exterior domains in which the acoustic pressure is written as an infinite modal sum. This method is similar in principle to that of Homicz and Lordi ${ }^{9}$, or Joseph and Morfey ${ }^{10}$; however, instead of looking for a direct transfer function between the interior and exterior modes, which is only available analytically for an unflanged circular duct, this method draws on the generality of the FEM. Thus, the modes in the interior and exterior domains are obtained numerically, which allows for the study of more general duct and flange geometries. This then delivers a hybrid method similar to that discussed by Kirby ${ }^{1}$, but here the hybrid method is used to join interior and exterior sound fields rather than two interior fields. This method also has the potential to retain the traditional banded symmetric matrix of the FEM, which can greatly speed up solution time ${ }^{1}$ 
and naturally avoids the use of artificial boundaries and PMLs in order to enforce the far field radiation boundary condition exactly.

A hybrid FE method joining interior and exterior domains is presented in Section II. Modal solutions for the duct and the exterior domain are found first, and then joined to a FE discretisation surrounding the end of the duct. Numerical calculations for duct end correction are then benchmarked against those classical works for flanged and unflanged circular ducts in Section III. The generality of the approach is explored by studying sound radiation from flanged and unflanged rectangular ducts since this presents a fully three dimensional problem that is also of practical interest. In fact it is interesting to note here that very little attention has been devoted in the literature to sound radiation from rectangular ducts, which is rather surprising given the widespread use of rectangular ducts in HVAC systems. Here, the HVAC community even records this lack of knowledge, for example ASHRAE ${ }^{27}$ quotes "It is not known whether [data based on circular ducts] can be accurately used with these diffusers [of high aspect ratio]"; that is, for rectangular ducts of high aspect ratio there is no evidence or data justifying the use of their current method, which is based on calculating an equivalent diameter for the rectangular duct and substituting this back into data measured for circular ducts. Accordingly, we seek here to address these fundamental issues and to present transmission loss regression formulae suitable for HVAC systems.

\section{THEORY}

The geometry of the duct is shown in Fig. 1, with a flange at an arbitrary angle $\alpha$, where $0^{\circ} \leq \alpha \leq 90^{\circ}$, and $\alpha=0^{\circ}$ corresponds to an unflanged duct, $\alpha=90^{\circ}$ to a flanged duct in 
which the length of the flange is considered to be infinite in all directions. In practice this means that the length of the flange is much larger than the wavelength of sound considered. A spherical co-ordinate system $r, \theta$ and $\phi$ is adopted where $\theta$ is the angle between the $z$ axis and the radius vector $r$, and $\phi$ is the angle between the $x$ axis and the projection of the radius vector $r$ onto the plane formed by the axes $x$ and $y$, where $x, y$ and $z$ form an orthogonal Cartesian co-ordinate system. Note also that Fig. 1 has been drawn in two dimensions for clarity; however, the model here is completely general so that the (uniform) cross section of $R_{1}$ is arbitrary and, in general, $R_{2}$ is a section from a sphere of radius $R$. Sound propagation in region $q(q=1,2$ or 3$)$ is governed by the acoustic wave equation

$$
\frac{1}{c_{q}^{2}} \frac{\partial^{2} p_{q}^{\prime}}{\partial t^{2}}-\nabla^{2} p_{q}^{\prime}=0
$$

where $c_{q}$ is the speed of sound, $p_{q}^{\prime}$ is the acoustic pressure, and $t$ is time. Equation (1) is solved in regions $R_{1}$ and $R_{3}$ by expanding the sound pressure as an infinite sum over eigenmodes in each region, whereas in $R_{2}$ a full finite element (FE) discretisation is used.

\section{A. Eigenvalue analysis}

For region $R_{3}$, the sound pressure field is expanded in the form

$$
p_{3}^{\prime}(r, \theta, \phi ; t)=\sum_{n=0}^{\infty} B_{n} \Upsilon_{n}(r) \Psi_{n}(\theta, \phi) \mathrm{e}^{i \omega t}
$$

Here, $B_{n}$ are the modal amplitudes, $i=\sqrt{-1}, \omega$ is the radian frequency, and it is assumed that the sound pressure field may be separated into a radial component $\Upsilon(r)$ and a transverse 
component $\Psi(\theta, \phi)$. The modal expansion in Eq. (2) has traditionally been applied in spherical harmonics $^{28}$, where it is usual to further separate the $\theta$ and $\phi$ components when examining sound radiation from a sphere. This expansion is still applicable to conical flanges, although one can no longer readily separate the $\theta$ and $\phi$ components and so these are combined here in the function $\Psi(\theta, \phi)$ in Eq. (2). Substitution of Eq. (2) into the governing wave equation for region $\mathrm{R}_{3}$ yields, after separating variables and re-arranging

$$
\frac{1}{\Upsilon_{n}(r)}\left[r^{2} \frac{\partial^{2} \Upsilon_{n}(r)}{\partial r^{2}}+2 r \frac{\partial \Upsilon_{n}(r)}{\partial r}\right]+k_{3}^{2} r^{2}=-\frac{1}{\Psi_{n}(\theta, \phi)} \nabla_{\theta \phi}^{2} \Psi_{n}(\theta, \phi),
$$

where the operator $\nabla_{\theta \phi}^{2}=\frac{1}{\sin \theta} \frac{\partial}{\partial \theta}\left(\sin \theta \frac{\partial}{\partial \theta}\right)+\frac{1}{\sin \theta} \frac{\partial^{2}}{\partial \phi^{2}}$.

Separation of variables in Eq. (3) means that both sides of this equation must equal a constant $\mathrm{C}$ (see Morse and $\operatorname{Ingard}^{28}$ ). If we first set $\mathrm{C}=\mathrm{s}_{n}^{2}$, then the right hand side of Eq. (3) may be written as

$$
\nabla_{\theta \phi}^{2} \Psi_{n}(\theta, \phi)+s_{n}^{2} \Psi_{n}(\theta, \phi)=0
$$

Similarly, if we choose $\mathrm{C}=\sigma_{n}\left(\sigma_{n}+1\right)$ for the left hand side of Eq. (3), then

$$
\zeta^{2} \frac{\partial^{2} \Upsilon_{n}(r)}{\partial \zeta^{2}}+2 \zeta \frac{\partial \Upsilon_{n}(r)}{\partial \zeta}+\left[\zeta^{2}-\sigma_{n}\left(\sigma_{n}+1\right)\right] \Upsilon_{n}(r)=0
$$

with $\zeta=k_{3} r$. Of course, we must also have 


$$
\mathrm{s}_{n}^{2}=\sigma_{n}\left(\sigma_{n}+1\right)
$$

Equation (5) is solved analytically, to give

$$
\Upsilon_{n}(r)=h_{\sigma_{n}}^{(2)}\left(k_{3} r\right)
$$

where $h_{\sigma_{n}}^{(2)}$ is spherical Hankel function of the second kind, of order $\sigma_{n}$. This is a well known result in spherical harmonics: for sound radiation from a sphere, symmetry permits the sound field to be expressed in terms of trigonometric functions for the $\phi$ variation, and Legendre functions for the $\theta$ variation $^{28}$, which delivers spherical Hankel functions of integer order, with $\sigma_{n}=0,1,2,3$ for a sphere. In the present study, sound radiates from a truncated sphere, or conical flange, and so the spherical Hankel functions are no longer of integer order (except for the fundamental mode where $\sigma_{n}=0$ ). Nevertheless, it is still possible first to solve Eq. (4) for $\Psi_{n}(\theta, \phi)$ and $s_{n}$, and then through the use of Eq. (6) to specify the radial dependence of the sound field in region $\mathrm{R}_{3}$ using spherical Hankel functions of non-integer order for the higher order modes.

The modal expansion chosen in Eq. (2) may readily be applied to a conical flange with $0<\alpha \leq 90^{\circ}$; however, in the limit $\alpha=0$ (an unflanged duct) one can no longer make use of a conical flange. To address this, the origin of the sphere $\left(\mathrm{R}_{2}\right)$ is placed on the plane $z=0$, so that the surface $\Gamma_{\mathrm{B}}$ is no longer perpendicular to the wall of the duct, see Fig. 2. This means that the projection of the sound pressure field from the surface $\Gamma_{B}$ into region $R_{3}$, using the modal expansion in Eq. (2), is unable to capture the sound pressure in the shaded region 
shown in Fig. 2. Thus, at a radius $r_{\mathrm{s}}$ the pressure over the arc length $\Delta \Gamma$ is omitted from the model. Therefore for unflanged ducts one must balance the size of region $\mathrm{R}_{2}$, which fully captures the sound pressure field, against the computational expenditure associated with making $R$ very large in order to minimise $\Delta \Gamma$ at a given radius $r_{\mathrm{s}}$. Of course, a similar problem also exists as $\alpha \rightarrow 0$ since it becomes very computationally inefficient to move the origin of the sphere - and hence to mesh a very large sphere - in order to accommodate very small values of $\alpha$. Clearly then Eq. (2) will only provide an approximate expansion for the sound pressure field in region $\mathrm{R}_{3}$ as $\alpha \rightarrow 0$; however, as $r$ increases in region $\mathrm{R}_{3}$ the pressure drops away proportional to $1 / r$ and so one may reasonably expect to be able to minimise this effect by carefully choosing the size of $\mathrm{R}_{2}$; this is explored further in section IIIA where results are reported for an unflanged duct.

In order to implement the hybrid method, Eq. (4) is first solved over the surface $\Gamma_{\mathrm{B}}$, which is the outer surface of the sphere that makes up the finite element mesh in region $R_{2}$, see Fig. 1 . Equation (4) is solved using the finite element method, where for mode $n$, the eigenfunction $\Psi(\theta, \phi)$ is approximated as

$$
\Psi(\theta, \phi)=\sum_{j=1}^{n_{3}} N_{3_{j}}(\theta, \phi) \Psi_{3_{j}},
$$

where $N_{3_{j}}$ is a global trial (or shape) function for the finite element mesh, $\Psi_{3_{j}}$ is the value of the eigenfunction at node $j$, and $n_{3}$ is the number of nodes on $\Gamma_{\mathrm{B}}$. Here, the finite element mesh, and hence nodal locations, for the surface $\Gamma_{\mathrm{B}}$ is taken from the surface of the mesh 
generated for region $\mathrm{R}_{2}$, noting that one must apply the appropriate coordinate

transformations when moving from a Cartesian $\left(\mathrm{R}_{2}\right)$ to a spherical coordinate system $\left(\mathrm{R}_{3}\right)$.

Expressing Eq. (8) in vector form yields

$$
\Psi(\theta, \phi)=\mathbf{N}_{3} \Psi_{3},
$$

where $\mathbf{N}_{\mathbf{3}}$ is a row vector and $\boldsymbol{\Psi}_{\mathbf{3}}$ is a column vector with the corresponding element values given in Eq. (8). Galerkin's method and a weak formulation are now used, with the test functions chosen to be equal to the trial functions. Thus, Eq. (4) is rewritten as:

$$
\left[\int_{\Gamma_{B}}\left[\nabla_{\theta \phi} \mathbf{N}_{\mathbf{3}}^{\mathbf{T}} \nabla_{\theta \phi} \mathbf{N}_{\mathbf{3}}-\mathrm{s}^{2} \mathbf{N}_{\mathbf{3}}^{\mathbf{T}} \mathbf{N}_{\mathbf{3}}\right] d \Gamma_{B}\right] \boldsymbol{\Psi}_{\mathbf{3}}=\mathbf{0},
$$

where $\nabla_{\theta \phi}=\frac{\partial \mathbf{N}_{3}}{\partial \theta} \widehat{\boldsymbol{\theta}}+\frac{1}{\sin \theta} \frac{\partial \mathbf{N}_{3}}{\partial \phi} \widehat{\boldsymbol{\phi}}$, and $\widehat{\boldsymbol{\theta}}$ and $\widehat{\boldsymbol{\phi}}$ are unit normals in the direction of $\theta$ and $\phi$, respectively. A surface integral over the perimeter of $\Gamma_{B}$ naturally arises from the weak formulation in Eq. (10) and here the boundary condition of zero normal particle velocity over this perimeter (which is the surface of the conical flange) has been enforced. Equation (10) is a standard eigenvalue problem and is solved to give an unordered list of $m_{3}$ eigenvalues, $\mathbf{s}_{n}$, and eigenvectors, $\Psi_{n}(\theta, \phi)$. On substitution of $s_{n}$ into Eq. (6), the $r$ dependence of the sound pressure field may then be calculated using Eq. (7). Note here that $s_{n}^{2}$ is always real (with $0 \leq \mathrm{s}_{n}^{2}<\infty$ ), and so Eq. (6) yields both positive and negative (but always real) values for $\sigma_{n}$ and here only the positive values are retained for the outward propagating modes.

In region $R_{1}$, the sound pressure is also expressed as a modal sum so that 


$$
p_{1}^{\prime}(x, y, z)=\sum_{n=0}^{\infty} F_{n} \Phi_{n}(x, y) e^{-i k_{1} \lambda_{n} z}+\sum_{n=0}^{\infty} A_{n} \Phi_{n}(x, y) e^{i k_{1} \lambda_{n} z}
$$

Here, $F_{n}$ and $A_{n}$ are the modal amplitudes, and $\lambda_{n}$ are the (dimensionless) wavenumbers in region $R_{1}$. In addition, $\Phi_{n}(x, y)$ are the eigenfunctions for the transverse plane $\left(\Gamma_{\mathrm{A}}\right)$ normal to the direction of wave propagation in $R_{1}$. A total of $n_{1}$ eigenvalues and eigenvectors are obtained following the same procedure as above, which is not reported here as it has appeared many times in the literature, see for example Kirby ${ }^{1}$. The incident sound field in this article is assumed to be planar, so that $F_{0}=1$ and $F_{n}=0$ for $n>0$, although one can readily include higher order modes in the incident duct, see for example Kirby and Lawrie ${ }^{29}$.

\section{B. Hybrid method}

The hybrid method follows the methodology of Kirby ${ }^{1}$, although here the method is used to join interior and exterior domains. Thus, for region $R_{2}$ the acoustic pressure is approximated in the same way as in the previous section, to give

$$
p_{2}(x, y, z)=\sum_{j=1}^{n_{2}} N_{2_{j}}(x, y, z) p_{2_{j}}=\mathbf{N}_{2} \mathbf{p}_{2}
$$

where $N_{2 j}$ is a global trial (or shape) function for the finite element mesh in region $R_{2}, p_{2_{j}}$ is the value of the sound pressure at node $j, n_{2}$ is the number of nodes in region $R_{2}$; and $\mathbf{N}_{2}$ and $\mathbf{p}_{2}$ are row and column vectors respectively. After applying Galerkin's method, the governing equation in region $R_{2}$ is written as 


$$
\left[\int_{R_{2}}\left[\nabla \mathbf{N}_{2}^{\mathbf{T}} \nabla \mathbf{N}_{2}-k_{2}^{2} \mathbf{N}_{2}^{\mathbf{T}} \mathbf{N}_{2}\right] d R_{2}\right] \mathbf{p}_{2}=\int_{\Gamma_{2}} \mathbf{N}_{2}^{\mathbf{T}} \nabla p_{2} \cdot \mathbf{n}_{2} d \Gamma_{2}
$$

Here, $\mathbf{n}_{2}$ is the outward unit normal vector to $R_{2}$. The hybrid method proceeds by enforcing continuity of acoustic pressure and normal velocity over $\Gamma_{A}$ and $\Gamma_{B}$ and these conditions are enforced here using mode matching. Note that mode matching enforces only continuity of pressure and continuity of axial velocity flux exactly, see for example the discussion by Lawrie and Guled ${ }^{30}$. Following Kirby ${ }^{1}$, continuity of pressure over $\Gamma_{\mathrm{A}}$ yields

$$
p_{2}(x, y,-L)=\sum_{n=0}^{m_{1}} F_{n} \Phi_{n}(x, y) e^{i k_{1} \lambda_{n} L}+\sum_{n=0}^{m_{1}} A_{n} \Phi_{n}(x, y) e^{-i k_{1} \lambda_{n} L}
$$

and continuity of velocity yields

$$
\frac{\partial p_{2}}{\partial z}(x, y,-L)=-i k_{1}\left\{\sum_{n=0}^{m_{1}} F_{n} \lambda_{n} \Phi_{n}(x, y) e^{i k_{1} \lambda_{n} L}-\sum_{n=0}^{m_{1}} A_{n} \lambda_{n} \Phi_{n}(x, y) e^{-i k_{1} \lambda_{n} L}\right\}
$$

Similarly, over $\Gamma_{B}$

$$
p_{2}(R, \theta, \phi)=\sum_{n=0}^{m_{3}} B_{n} \Upsilon_{n}(R) \Psi_{n}(\theta, \phi)
$$

and

$$
\frac{\partial p_{2}}{\partial r}(R, \theta, \phi)=\sum_{n=0}^{m_{3}} B_{n} \Upsilon_{n}^{\prime}(R) \Psi_{n}(\theta, \phi)
$$

where $\Upsilon_{n}^{\prime}$ denotes the derivative of $\Upsilon_{n}$ with respect to $r$. Here, it has been assumed that the same fluid is present in each region and that the infinite sums are truncated at $m_{1}$ and $m_{3}$ in regions $R_{1}$ and $R_{3}$, respectively. By assuming Neumann boundary conditions over the surfaces of the duct and flange, the surface integral in Eq. (13) may be written as 


$$
\begin{gathered}
\int_{\Gamma_{2}} \mathbf{N}_{2}^{\mathbf{T}} \nabla p_{2} \cdot n_{2} d \Gamma_{2}=i k_{1} \int_{\Gamma_{A}} \mathbf{N}_{2}^{\mathbf{T}} \sum_{n=0}^{m_{1}}\left[F_{n} \lambda_{n} \Phi_{n}(x, y) e^{i k_{1} \lambda_{n} L}-A_{n} \lambda_{n} \Phi_{n}(x, y) e^{-i k_{1} \lambda_{n} L}\right] d \Gamma_{A} \\
+\int_{\Gamma_{B}} \mathbf{N}_{2}^{\mathbf{T}} \sum_{n=0}^{m_{3}} B_{n} \Upsilon_{n}^{\prime}(R) \Psi_{n}(\theta, \phi) d \Gamma_{B} .
\end{gathered}
$$

Equation (18) thus enforces the velocity matching conditions; the pressure matching conditions are enforced separately and, after weighting each condition and integrating ${ }^{1}$, for $\Gamma_{\mathrm{A}}$ this gives

$$
\begin{aligned}
i k_{1} \lambda_{m} \int_{\Gamma_{A}} \Phi_{m} \mathbf{N}_{\mathbf{2}} d \Gamma_{A} \mathbf{p}_{\mathbf{2 A}}= \\
\quad i k_{1}\left[\sum_{n=0}^{m_{1}} \lambda_{m} F_{n} e^{i k_{1} \lambda_{n} L} \int_{\Gamma_{A}} \Phi_{m} \Phi_{n} d \Gamma_{A}+\sum_{n=0}^{m_{1}} \lambda_{m} A_{n} e^{-i k_{1} \lambda_{n} L} \int_{\Gamma_{A}} \Phi_{m} \Phi_{n} d \Gamma_{A}\right]
\end{aligned}
$$

Similarly, for $\Gamma_{\mathrm{B}}$

$$
\int_{\Gamma_{B}} \Psi_{m} \mathbf{N}_{\mathbf{2}} d \Gamma_{B} \mathbf{p}_{2 \mathbf{B}}=\sum_{n=0}^{m_{3}} B_{n} \Upsilon_{n}(R) \int_{\Gamma_{B}} \Psi_{m} \Psi_{n} d \Gamma_{B}
$$

Here, $\mathbf{p}_{\mathbf{2 A}}$ and $\mathbf{p}_{\mathbf{2 B}}$ denote values of the finite element solution in region $R_{2}$ at the nodal locations on the surfaces $\Gamma_{\mathrm{A}}$ and $\Gamma_{\mathrm{B}}$, respectively. Note that in weighting Eqs. (19) and (20), the eigenfunction $\Phi_{m}$ is the transpose of $\Phi_{n}$, and $\Psi_{m}$ is the transpose of $\Psi_{n}$. The problem may now be expressed in matrix form to give

$$
\mathbf{G p}_{2}+\mathbf{Q}_{1}^{\mathrm{T}} \widetilde{\mathbf{A}}-\mathbf{Q}_{3}^{\mathrm{T}} \widetilde{\mathbf{B}}=\mathbf{Q}_{1}^{\mathrm{T}} \widetilde{\mathbf{F}}
$$




$$
\begin{gathered}
Q_{1} p_{2 A}-M_{1} \widetilde{A}=M_{1} \widetilde{\mathbf{F}} \\
-Q_{3} p_{2 B}+M_{3} D_{3}^{-1} \widetilde{B}=0 .
\end{gathered}
$$

where $\mathbf{D}_{\mathbf{1}}$ and $\mathbf{D}_{\mathbf{3}}$ are diagonal matrices with each diagonal element given by $e^{-i k_{1} \lambda_{n} L}$, $\left(n=0,1, \cdots, m_{1}\right)$, and $\Upsilon_{n}^{\prime}(R),\left(n=0,1, \cdots, m_{3}\right)$, respectively, with $\mathbf{D}_{\mathbf{1}} \mathbf{A}=\widetilde{\mathbf{A}}, \mathbf{D}_{3} \mathbf{B}=\widetilde{\mathbf{B}}$ and $\mathbf{F}=\mathbf{D}_{\mathbf{1}} \tilde{\mathbf{F}}$. In addition,

$$
\begin{gathered}
\mathbf{G}=\int_{\Omega_{2}}\left[\nabla \mathbf{N}_{2}^{\mathrm{T}} \nabla \mathbf{N}_{2}-k_{2}^{2} \mathbf{N}_{\mathbf{2}}^{\mathrm{T}} \mathbf{N}_{2}\right] d \Omega_{2} \\
\mathbf{Q}_{\mathbf{1}}=i k_{1} \lambda_{m} \int_{\Gamma_{A}} \Phi_{m} \mathbf{N}_{\mathbf{2}} d \Gamma_{A} \quad\left(m=0,1, \cdots, m_{1}\right) \\
\mathbf{Q}_{\mathbf{3}}=\int_{\Gamma_{B}} \Psi_{m} \mathbf{N}_{\mathbf{2}} d \Gamma_{B} \quad\left(m=0,1, \cdots, m_{3}\right) \\
\mathbf{M}_{\mathbf{1}}=i k_{1} \lambda_{m} \int_{\Gamma_{A}} \Phi_{m} \Phi_{n} d \Gamma_{A} \quad\left(m=0,1, \cdots, m_{1} ; n=0,1, \cdots, m_{1}\right) \\
\mathbf{M}_{\mathbf{3}}=\Upsilon_{n}(R) \int_{\Gamma_{B}} \Psi_{m} \Psi_{n} d \Gamma_{B} \quad\left(m=0,1, \cdots, m_{3} ; n=0,1, \cdots, m_{3}\right)
\end{gathered}
$$

To combine Eqs. (21)-(23), the matrix $\mathbf{G}$ is divided up to give

$$
\mathbf{G p}_{2}=\left[\begin{array}{lll}
\mathbf{G}_{2 A A} & \mathbf{G}_{2 A e} & \mathbf{G}_{2 A B} \\
\mathbf{G}_{2 \mathrm{eA}} & \mathbf{G}_{2 \mathrm{ee}} & \mathbf{G}_{2 \mathrm{eB}} \\
\mathbf{G}_{2 \mathrm{BA}} & \mathbf{G}_{2 \mathrm{Be}} & \mathbf{G}_{2 \mathrm{BB}}
\end{array}\right]\left\{\begin{array}{l}
\mathbf{p}_{2 \mathrm{~A}} \\
\mathbf{p}_{2 \mathrm{e}} \\
\mathbf{p}_{2 \mathrm{~B}}
\end{array}\right\}
$$

The problem is then assembled into its final matrix form, to give 


$$
\left[\begin{array}{ccccc}
-\mathbf{M}_{1} & \mathbf{Q}_{1} & \mathbf{0} & \mathbf{0} & \mathbf{0} \\
\mathbf{Q}_{1}^{\mathrm{T}} & \mathbf{G}_{2 \mathrm{AA}} & \mathbf{G}_{2 \mathrm{Ae}} & \mathbf{G}_{2 \mathrm{AB}} & \mathbf{0} \\
\mathbf{0} & \mathbf{G}_{2 \mathrm{eA}} & \mathbf{G}_{2 \mathrm{ee}} & \mathbf{G}_{2 \mathrm{eB}} & \mathbf{0} \\
\mathbf{0} & \mathbf{G}_{2 \mathrm{BA}} & \mathbf{G}_{2 \mathrm{Be}} & \mathbf{G}_{2 \mathrm{BB}} & -\mathbf{Q}_{3}^{\mathrm{T}} \\
\mathbf{0} & \mathbf{0} & \mathbf{0} & -\mathbf{Q}_{3} & \mathbf{D}_{3}^{-1} \mathbf{M}_{3}
\end{array}\right]\left\{\begin{array}{c}
\widetilde{\mathbf{A}} \\
\mathbf{p}_{2 \mathrm{~A}} \\
\mathbf{p}_{2 \mathrm{e}} \\
\mathbf{p}_{2 \mathrm{~B}} \\
\widetilde{\mathbf{B}}
\end{array}\right\}=\left\{\begin{array}{c}
\mathbf{M}_{\mathbf{1}} \tilde{\mathbf{F}} \\
\mathbf{Q}_{1}^{\mathrm{T}} \tilde{\mathbf{F}} \\
\mathbf{0} \\
\mathbf{0} \\
\mathbf{0}
\end{array}\right\} .
$$

Equation (30) is a set of $n_{t}\left(=m_{1}+n_{2}+m_{3}\right)$ linear equations, where $n_{2}$ is the number of nodes in region $R_{2}$, and $m_{1}$ and $m_{3}$ are the number of modes in regions $R_{1}$ and $R_{3}$, respectively. Thus this method retains the advantages described by Kirby ${ }^{1}$, such as a sparse, symmetric, banded matrix where values for $m_{1}$ and $m_{3}$ can also be minimised to reduce computational expenditure. Modal amplitudes and the acoustic pressures in $R_{2}$ are then found on the solution of Eq. (30), thus fully describing the pressure distribution in the interior and exterior domains.

Results are presented here for the duct end correction and the duct transmission loss (TL). The reflection coefficient for a plane incident wave in $R_{1}$ is given by, $\Lambda_{0}=A_{0} / F_{0}$. The length correction is defined by referring to a duct with an 'effective' length that terminates with zero impedance ${ }^{5}$. Thus, the end correction $\delta$ is related to the reflection coefficient by the following expression

$$
\Lambda_{0}=-\left|\Lambda_{0}\right| e^{-2 i k_{1} \delta}
$$

The transmission loss is the ratio of incident and transmitted sound powers, which is given as

$$
\mathrm{TL}=-10 \log _{10}\left[\frac{1}{k_{1} k_{3}} \frac{\sum_{n=0}^{n_{t r}} \mathrm{H}_{n}\left|B_{n}\right|^{2}}{\sum_{n=0}^{n_{i n c}} \lambda_{n} \mathrm{I}_{n}\left|F_{n}\right|^{2}}\right] .
$$


where $n_{\text {inc }}$ and $n_{t r}$ denote the number of cut-on modes for the incident and transmitted waves, respectively; and $\mathrm{I}_{n}=\int_{\Gamma_{A}}\left|\Phi_{n}(x, y)\right|^{2} d \Gamma_{\mathrm{A}}$ and $\mathrm{H}_{n}=\int_{\Gamma_{\mathrm{B}}}\left|\Psi_{n}(\theta, \phi)\right|^{2} d \Gamma_{\mathrm{B}}$.

\section{RESULTS AND DISCUSSION}

\section{A. Circular ducts}

The numerical model described in the previous section is validated first by comparing predictions with analytic and numerical results reported in the literature for two classical cases: a flanged $\left(\alpha=90^{\circ}\right)$ and unflanged $\left(\alpha=0^{\circ}\right)$ circular duct. For both cases, the axisymmetric geometry allows the FE model described in Section II to be reduced to two dimensions, which speeds up computation time. In the calculations that follow, the fluid in all three regions is assumed to be air, in which the speed of sound $c=343.2244 \mathrm{~m} / \mathrm{s}$, the duct radius $a=50 \mathrm{~mm}$, and different values for the non-dimensional variable $k a$ have been obtained by changing only the frequency. For the finite element discretisation of $R_{2}$, eight noded quadrilateral isoparametric elements are used in the interior domain, and six noded triangular isoparametric elements are used for the exterior. A minimum of sixteen nodes per wavelength are used here in order to deliver confidence in the accuracy of each solution, although in the study of convergence that follows (see Table I), the number of nodes may be increased.

Before comparing the FE predictions with those reported in the literature, it is important first to establish the relative convergence of the FE method. In view of previous studies ${ }^{1,31}$, it is prudent to investigate how the locations of $\Gamma_{A}$ and $\Gamma_{B}$ influence the accuracy and convergence 
of the hybrid method. Of course, the locations of $\Gamma_{A}$ and $\Gamma_{B}$ also affect computational cost since they directly influence the number of nodes in $R_{2}$; however, one cannot simply locate these surfaces very close to the duct aperture since in regions of strong sound scattering it becomes progressively more difficult to represent accurately the (more complex) sound pressure field in terms of a modal expansion. Accordingly, the aim here is to establish a compromise between accuracy and speed of the solution, although the emphasis in this paper will be placed on accuracy in view of the regression formulae presented later on. The locations of $\Gamma_{A}$ and $\Gamma_{B}$ are defined in Fig. 1 by the dimensions $L$ and $R$, respectively. In Table I values for the end correction coefficient $\delta / a$ are compared for a flanged duct with different values of $L$ and $R$ for Helmholtz numbers of $k a=0.1$ and $k a=1$. Here, a very fine FE mesh is used in $R_{2}$, with approximately 300 nodes per wavelength so that when, for example, $k a=1, L / a=2$ and $R / a=2$ the number of nodes $n_{2}=13096$; the number of modes in the interior domain and the exterior domain are both set equal to $10\left(m_{1}=m_{3}=10\right)$ for $k a \leq 1$.

To investigate optimum locations for $\Gamma_{A}$ and $\Gamma_{B}$, the surface $\Gamma_{B}$ is fixed first with a value of $R / a=2$; the surface $\Gamma_{A}$ is then progressively moved away from the duct aperture. It can be seen in Table I that the location of $\Gamma_{A}$ makes very little difference to the end correction $\delta / a$, although a value of $L / a=0.2$ appears to be an acceptable compromise given that only one element is required within the duct, which incurs negligible computational cost. Next, $\Gamma_{A}$ is fixed and $\Gamma_{B}$ is moved away from the duct aperture; here, a value of $L / a=1$ is chosen for $\Gamma_{A}$ to ensure that the influence of $\Gamma_{B}$ is isolated. It is evident in Table I that an improvement in accuracy can again be achieved by moving the boundary away from the duct aperture. Furthermore, since the element size is pre-determined according to wavelength, a larger radius $R$ implies a higher element density along $\Gamma_{B}$, which improves the accuracy of the 
numerical integration over $\Gamma_{B}$. Of course, the total number of nodes in $R_{2}$ is proportional to $R^{2}$ and so any improvement in accuracy as $R$ is increased is at the expense of a significant increase in computational expenditure. However, a value of $R / a=2$ is able to obtain results converged up to 6 decimal places, which is enough for engineering applications. In Table II data is presented for $\Gamma_{B}$ for an unflanged circular duct $\left(\Gamma_{A}\right.$ follows very similar behaviour to that seen in Table I) and here the convergence is still acceptable, although slightly slower than that seen for the flanged duct and convergence to three decimal places requires a value of $R / a=4$. It is likely that the slower rates of convergence seen for the unflanged duct are caused by the approximate nature of the modal expansion (see Fig. 2), although it is noted here that convergence is still relatively good and so that in practice results obtained with $R / a=2$ should be acceptable.

In view of the results presented in Tables I and II, and after undertaking many additional numerical experiments, compromise values of $L / a=0.2$ and $R / a=2$ are proposed here for the locations of $\Gamma_{A}$ and $\Gamma_{B}$, respectively, in an flanged duct, and $L / a=0.2$ and $R / a=4$ for a unflanged duct. This is aimed at prioritising prediction accuracy over solution speed, although it is possible to further reduce the value of $R / a$ if one is not so concerned with accuracy, which may be an acceptable approach if computing duct TL since this parameter is less sensitive to inaccuracies. Furthermore, it is also assumed that the influence of the location of $\Gamma_{A}$ and $\Gamma_{B}$ is consistent across all the calculations that follow in Section III. This assumption is based on a number of additional numerical experiments for both flanged and unflanged ducts that are not reported here, and in all future calculations values of $L / a=0.2$ and $R / a=2$ are adopted in order to balance accuracy and computational expenditure for the three dimensional analysis of rectangular ducts. 
Following the optimisation described above, the length correction coefficient $\delta / a$ for a flanged circular duct is presented in Fig. 3. Here, the axisymmetric geometry and the values chosen for $L$ and $R$ deliver a final system of equations with 9651 degrees of freedom, which takes about one second to solve for each frequency (using MATLAB on a $2.93 \mathrm{GHz}$ Intel Core $^{\mathrm{TM}} \mathrm{CPU}$ with a $8 \mathrm{~Gb}$ RAM). In Fig. 3 the FE predictions are compared with the analytic solution of Nomura et al. ${ }^{15}$, as well as the data fitting formulae of Silva et al. ${ }^{6}$ and Norris and Sheng ${ }^{13}$. The analytic solutions and the data fitting formulae both assume plane wave propagation in the duct and so are valid for $k a<3.832$, whereas the FE model is extended here to $k a=5$ by including higher order reflected modes in the duct, although only plane wave excitation is considered. Silva et al. produced three different models for calculating the duct end correction coefficient; however, in order to satisfy causality and Hermitian symmetry, the accuracy of their first two models is compromised and so we only report their third model here since this gives their most accurate result. It is evident in Fig. 3 that very good agreement between all models is observed when $k a<3$, although the FE model is closer to the analytic model of Nomura et al. (which overlays the FE solution) when compared to the data fitting formulae. Over the frequency range $0.5<k a<2$, Silva et al.'s data fitting formulae slightly underestimates $\delta / a$, while Norris and Sheng's slightly overestimate $\delta / a$. In fact, the FE solution and Nomura et al.'s analytic predictions agree to within $1.7 \%$ of one another up to $k a=3.5$, and they also appear to lie between the two data fitting formulae. Clearly, the data in Fig. 3 provides convincing evidence that the FE model developed here is capable of delivering accurate predictions. 
The end correction coefficient for an unflanged circular duct is presented in Fig. 4. In view of the limitations of the modal expansion used in Eq. (2) (shown in Fig. 2) this example is a more rigorous test of the convergence of the numerical model. Following on from the optimisation procedure for a flanged duct, values of $m_{1}=10, m_{3}=30$ and a total of 63990 degrees of freedom are chosen here. Predictions are compared with the analytic solution of Levine and Schwinger ${ }^{3}$ in Fig. 4, as well as the data fitting formulae of Silva et al. ${ }^{6}$ and Dalmont et al. ${ }^{5}$. Again we use Silva et al.'s most accurate model, but here Dalmont et al.'s data fitting formula is used since this is based on formulae presented by Norris and Sheng ${ }^{13}$ but with the addition of a corrective term to improve accuracy when $k a<1.5$. In Fig. 4 , Levine and Schwinger's analytic solution is seen to overlay the FE model and the difference between the two predictions is less than $1.3 \%$ for $k a<3$. This clearly demonstrates that, even for the limiting case of $\alpha=0$, the modal expansion for the radiating sound field is capable of accurately capturing the physics of the problem. This is because the contribution of the sound pressure field from the region in the "shadow" of the duct to overall duct performance is low, especially close to the duct wall in region $\mathrm{R}_{3}$, and so the effect of omitting a small portion of this region from the model (the shaded region in Fig. 2) is negligible. Moreover, in this region the decay of the sound pressure field is proportional to $1 / r$, which serves to further reduce the influence of the shaded area on the duct characteristics. Thus, for $\alpha=0$, and $\alpha \rightarrow 0$ it appears sensible to place the centre of region $\mathrm{R}_{2}$ at $z=0$ in order to minimise computational expenditure, although the precise point at which one should move away from this strategy when $\alpha>0$ will be apparent only after conducting parametric studies for particular duct geometries and frequencies of interest. It is not surprising to note that the data fitting formulae are not so accurate over the whole frequency range in Fig. 4, and for $1.8<k a<2.8$, Silva et al.'s and Dalmont et al.'s data fitting formulae are seen to deviate from the FE predictions. The purpose here, however, is to 
validate the FE model and it is clear from the results in Figs. 3 and 4 that excellent agreement between the FE predictions and benchmark analytic models has been obtained for a simple axisymmetric geometry and at limiting angles for the flange. Furthermore, the generality of the method allows for an extension of these predictions to much higher values of $k a$, since the method may readily accommodate both incident and reflected higher order modes within the duct.

In Figs. 3 and 4 predictions are presented using the duct end correction, which is the traditional method for quantifying sound radiation from the open end of a duct; however, the acoustic design of industrial ducting systems that contain noise sources such as fans (e.g. HVAC systems), normally quantify acoustic performance in terms of a sound power balance such as sound transmission loss (TL), see Eq. (32). Here, a simple sound power balance also offers a further method of validating the FE model and, on balancing incident, reflected and transmitted sound powers, a maximum error of $10^{-12} \%$ was obtained for an unflanged duct, which equates to machine accuracy. This indicates that the model is conserving energy, although Lawrie and Kirby ${ }^{32}$ caution against using only a simple power balance to indicate that a model has been implemented correctly. Of more interest is the duct TL and in Fig. 5 the TL for a flanged and unflanged circular duct is reported, based on the application of Eq. (32). It is not surprising to note here that the TL for an unflanged duct is larger than that for a flanged duct over the entire range of $k a$, indicating that the unflanged duct radiates less sound power into free space. This is because of the impedance mismatch over the duct aperture being lowered by the presence of a flange. It is noticeable that for low values of $k a$, say $k a<0.5$, a significant amount of sound power is reflected back into the duct for both termination conditions. This effect is well known and provides considerable assistance when attenuating low frequency fan noise in ducting systems. At higher values of $k a$ the TL 
reduces significantly and, for $k a>2, T L<1 \mathrm{~dB}$, which indicates that the end of the duct has little practical influence on sound propagation at higher frequencies. Moreover, above the first cut-on frequency, $k a>3.832$, very little change in the TL is observed and so it appears acceptable for engineering purposes to neglect the TL for $k a>2$. Thus, in Fig. 5 it is demonstrated that for circular ducts the influence of the end of the duct on sound propagation needs only to be included for plane wave propagation within the duct. This facilitates the inclusion of the duct TL in simple design procedures for HVAC systems and in the UK $\mathrm{CIBSE}^{33}$ presents the following regression formula for a flanged circular duct:

$$
T L_{\mathrm{CIBSE}}=10 \log _{10}\left[1+\left(\frac{0.8}{k a}\right)^{1.88}\right]
$$

In the U.S., ASHRAE ${ }^{27}$ present numerical data for different duct diameters, however if one plots this data then it is seen that Eq. (33) is simply a regression curve fitted through the data presented by ASHRAE. Thus, both professional bodies provide very similar predictions of duct performance, which is not surprising since they are largely based on the same set of experimental data. It is interesting, therefore, to obtain a regression curve for the theoretical predictions presented in Fig.5. For an unflanged circular duct this gives

$$
T L=10 \log _{10}\left[1+\frac{1}{(k a)^{2}}\right]
$$

and for a flanged circular duct

$$
T L=10 \log _{10}\left[1+\frac{1}{2(k a)^{2}}\right]
$$

where $a$ is the duct radius and $k$ the wavenumber. It is noticeable here that the theoretical values are different to those quoted by CIBSE, however for a flanged duct the difference 
between Eqs. (33) and (35) is less than $0.6 \mathrm{~dB}$ for the range $0.06<k a<3.83$; only at very low $k a$ values do the two models begin to depart significantly (where it is likely that significant errors will be present in the experimental data). Furthermore, the difference between Eqs. (34) and (35), and their respective FE solutions over the entire frequency range is less than $0.25 \mathrm{~dB}$ for the flanged duct, and less than $0.42 \mathrm{~dB}$ for the unflanged duct. Here, these formulae have been chosen to provide very good correlation with the FE model at lower $k a$ values where the influence on system performance is most significant. Note also that Eq. (37) is very close to the values that may be obtained from the analytic study of Levine and Schwinger ${ }^{3}$.

\section{B. Rectangular ducts}

In the previous section the FE model was validated against classical solutions for a simple axisymmetric duct geometry. Of course, the real purpose of developing a more general model is to examine more complex duct geometries. Accordingly, sound radiation from a rectangular duct is examined here since this presents a fully three dimensional problem whilst also having some practical interest for HVAC systems. Following the previous section, both the end correction coefficient and TL are computed, but this is done for rectangular ducts with varying aspect ratios. Assuming the length of the longer side of the rectangular duct is denoted by $2 a$, and the shorter side by $2 b$, then the aspect ratio of the duct is defined as $H=a / b$, so that $H \geq 1$. According to European Standards ${ }^{34}$, a maximum value of $H=4$ is recommended for rectangular ductwork and so in this section the study of rectangular ducts is limited to the range $1 \leq H \leq 4$. Clearly, a rectangular geometry requires a fully 3D finite element discretisation of region $R_{2}$ and here six noded triangular isoparametric elements are used on the surface of region $R_{2}$, and ten noded tetrahedral isoparametric elements are used 
to mesh the volume of $R_{2}$. Following the previous section, we again set $L / a=0.2$ and $R / a=2$. To maintain accuracy but to economise on element numbers, the element size within $R_{2}$ is optimised so that it is finer on the surfaces $\Gamma_{\mathrm{A}}$ and $\Gamma_{\mathrm{B}}$ and coarser within the volume of $R_{2}$ in order to improve the accuracy of the integration over each surface. Here, at least 27 nodes per wavelength are used on each surface, whilst a minimum of 11 nodes per wavelength are used within the volume. Furthermore, as the aspect ratio of the duct $H \rightarrow 4$, additional elements are placed along the shorter cross-sectional dimension in view of the high pressure gradients that appear as $H$ increases. Thus, as $H$ increases it is necessary to increase the number of elements in the finite element discretisation. The examination of a rectangular duct also significantly increases the number of degrees of freedom required when compared to the circular duct studied previously, for example when $H=4$ a total of 81,437 nodes were used in region $R_{2}$ for a flanged rectangular duct, with $m_{1}=20$ and $m_{3}=120$. However, the hybrid method delivers a sparse, banded matrix, and it is possible to economise on allocated computer memory provided one takes the precaution of storing only the non-zero elements of the matrix. In this particular example, results for a single frequency took about three minutes on a $2.93 \mathrm{GHz}$ Intel Core ${ }^{\mathrm{TM}} \mathrm{CPU}$ with a $8 \mathrm{~Gb}$ RAM computer. Note that prior to generating predictions for rectangular ducts and assigning the mesh parameters described above, a three dimensional model was generated for a circular duct in order to benchmark predictions against the two dimensional model described in Section IIIA. Here, a maximum difference of $4 \%$ was observed between the end correction predicted by the two and three dimensional models over the range $0<k a \leq 4$. It is possible to lower this error further by increasing the number of elements in the three dimensional model; however, this would incur a very large increase in computational expenditure and so it was decided that a difference of $4 \%$ represented an acceptable compromise for the three dimensional model. It was noticeable 
also that, when conducting a power balance, the accuracy of the three dimensional model is comparable to that seen for the two dimensional model.

In Figs. 6 and 7 the end correction coefficients are presented for flanged and unflanged rectangular ducts, with aspect ratios of 1,2,3 and 4. The end corrections are plotted against $k a$, where for a rectangular duct $2 a$ is the length of the longest side. It is evident in both figures that the end correction drops as the aspect ratio increases. If we denote the mode number in the rectangular duct as $(m, n)$, where $m$ is the mode number along the longer side of the duct and $n$ is the mode number along the shorter side of the duct, then for a square duct identical mode shapes will exist in pairs. For example, the $(1,0)$ mode and $(0,1)$ mode will appear together after the first cut-on frequency $(k a=\pi / 2)$ and the superposition of these modes will make the acoustic field in a square duct similar to that in a circular duct. This partially explains the similarity between the end correction for the square duct and the circular duct, also plotted in Figs. 6 and 7. Note also that modes $(1,0)$ and/or $(0,1)$ have little influence on the end correction coefficient for a square duct, whereas the $(2,0)$ and $(0,2)$ modes significantly change the behaviour when $k a>\pi$. For the $(2,0)$ and/or $(0,2)$ mode, the pressure variation in the cross-sectional area of the duct is one wavelength, which resembles the first radial cut on mode in a circular duct at $k a=3.832$. For rectangular ducts with an aspect ratio larger than one, this behaviour becomes less pronounced since at $k a=\pi$, only the $(2,0)$ mode is cut-on. Therefore, the end correction does not drop as significantly as that of a square duct and the $(0,2)$ mode will be excited later, at a frequency $k a=H \pi$. It is clear in Figs. 6 and 7 that the end correction for a flanged rectangular duct is greater than that seen for an unflanged duct, which follows the pattern seen for circular ducts. Furthermore, as the aspect ratio increases the end correction drops at lower values of $k a$. In Figs. 6 and 7, the 
larger dimension $a$ is used for the Helmholtz number and this means that as $H$ increases $a$ is held constant and $b$ is reduced. Thus, the end correction is seen to drop as the area of the duct reduces.

In the previous section it was noted that for engineering purposes it is convenient to present data in terms of the duct TL. Accordingly, in Fig. 8 predictions are presented for a flanged rectangular duct with aspect ratios of 1,2,3 and 4. Here, it is interesting also to compare predictions against those values estimated by $\mathrm{CIBSE}^{33}$, who adapt their formulae for circular ducts by using an equivalent diameter $d$, where $d=\sqrt{4 A / \pi}$ and $A$ is the cross-sectional area of the rectangular duct. This gives

$$
T L_{\mathrm{CIBSE}}=10 \log _{10}\left[1+\left(\frac{0.4}{k a} \sqrt{\pi H}\right)^{1.88}\right]
$$

In Fig. 8 it is clear that the predictions of CIBSE are good for the square duct given the assumption of an equivalent diameter. Here, the maximum difference between the FE predictions and CIBSE's data is less than $0.7 \mathrm{~dB}$ for $0.025<k a<4$. Furthermore, for rectangular ducts with an aspect ratio greater than one, Eq.(36) maintains good agreement with the FE predictions, and for $H=4$ the difference between the two is still less than $1 \mathrm{~dB}$ for $0.025<k a<4$. Uncertainty regarding the accuracy of the TL values reported by CIBSE for rectangular ducts is well known and in addition $\mathrm{ASHRAE}^{27}$ note that "It is not known whether [data based on circular ducts] can be accurately used....[at high aspect ratios]". The results presented here clearly show that the formulae presented by ASHRAE and CIBSE provide good agreement with the FE predictions and so one can probably conclude that the 
data currently being quoted by CIBSE and ASHRAE provide a good approximation of actual duct performance.

To facilitate the straightforward application of the FE results, new regression formula for rectangular ducts with different aspect ratios are reported here. For an unflanged rectangular duct

$$
T L=10 \log _{10}\left[1+\frac{0.3909 H}{(k a)^{2}}\right]
$$

and for a flanged rectangular duct

$$
T L=10 \log _{10}\left[1+\frac{0.7296 H^{1.048}}{(k a)^{2}}\right]
$$

where the rectangular duct has dimensions $2 a \times 2 b$, and $H=a / b, \geq 1$. Equations (37) and (38) are aimed at accurately reproducing the FE predictions at low values of $k a$ and so they have been optimised for $k a<3$. Here, the maximum difference between the FE predictions and these regression formulae is $0.25 \mathrm{~dB}$ for $k a<3$ and $1 \leq H \leq 4$, moreover this error drops significantly as $k a \rightarrow 0$.

\section{CONCLUSIONS}

A hybrid numerical method is reported here that joins interior and exterior domains in order to analyse the radiation of sound from the open end of circular and rectangular ducts. The method relies on a full finite element discretisation of the region surrounding the open end of the duct. Here, values of $L / a=0.2$ and $R / a=2$ are recommended as optimum locations for 
the interface between the finite element discretisation and the modal expansions used in the interior and exterior regions, respectively. The hybrid method then allows one to economise on the number of degrees of freedom required, as well as avoiding the computational cost and inaccuracies associated with techniques such as PMLs, which are traditionally used with the finite element method for this type of problem.

The hybrid method is validated by comparing predictions of duct end correction against analytic predictions available in the literature for flanged and unflanged circular ducts. Here, excellent agreement between the different methods is observed, which demonstrates the ability of the hybrid method to deliver accurate predictions for duct radiation problems. Moreover, it is seen that the modal expansion proposed in Eq. (2) is capable of accurately representing the radiated sound pressure field, even for the limiting case of an unflanged duct. This provides confidence when extending the model to three dimensional problems, and here a comparison between TL predictions and experimental data ${ }^{27,33}$ reveals generally good agreement. Moreover, this directly addresses questions raised by both $\operatorname{CIBSE}^{27}$ and AHSRAE $^{33}$ regarding the accuracy of their data/formulae at larger values of $H$, whereby the results generated here serve to provide confidence in those values currently being used.

The predictions presented here demonstrate the flexibility and generality of the hybrid method and show that this method can readily be used to join interior and exterior problems. Of course, as the problem moves from two to three dimensions, a significant additional computational cost is incurred; however, the method implemented successfully minimises computational expenditure to the extent that the calculations in this article were run on a single desktop within an acceptable timescale. This is an advantage of the hybrid method, 
which for this problem retains the sparse, symmetric and banded matrix traditionally seen in the finite element method. This offers the possibility of using this type of model in an iterative design environment, although it is, of course, more convenient to use regression formulae, which is why they have also been reported here. Furthermore, the generality of the model presented provides a technique for studying more complex problems, such as more complicated duct/flange geometries and/or surfaces of finite impedance.

\section{ACKNOWLEDGEMENTS}

The authors would like to thank the UK Engineering and Physical Sciences Research Council (EPSRC) for their support of the work reported in this article. 


\section{REFERENCES}

1. R. Kirby, "Modeling sound propagation in acoustic waveguides using a hybrid numerical method," J. Acoust. Soc. Am. 124(4), 1930-1940 (2008).

2. Lord Rayleigh, The theory of sound, (Dover, New York, 1945) vol. II, chapter 16.

3. H. Levine and J. Schwinger, "On the radiation of sound from an unflanged circular pipe," Phys. Rev. 73(4), 383-406 (1948).

4. P. Davies, J. L. B. Coelho, and M. Bhattacharya, "Reflection coefficients for an unflanged pipe with flow," J. Sound Vib. 72(4), 543-546 (1980).

5. J. P. Dalmont, C. J. Nederveen, and N. Joly, "Radiation impedance of tubes with different flanges: Numerical and experimental investigations," J. Sound Vib. 244(3), 505-534 (2001).

6. F. Silva, P. Guillemain, J. Kergomard, B. Mallaroni, and A. N. Norris, "Approximation formulae for the acoustic radiation impedance of a cylindrical pipe," J. Sound Vib. 322(12), 255-263 (2009).

7. Y. Ando, "On the sound radiation from semi-infinite circular pipe of certain wall thickness," Acustica 22, 219-225 (1969).

8. M. Bernard and B. Denardo, "Re-computation of Ando's approximation of the end correction for a radiating semi-infinite circular pipe," Acustica 82(4), 670-671 (1996).

9. G. F. Homicz and J. A. Lordi, "A note on the radiative directivity patterns of duct acoustic modes,” J. Sound Vib. 41(3), 283-290 (1975).

10. P. Joseph and C. L. Morfey, "Multimode radiation from an unflanged, semi-infinite circular duct," J. Acoust. Soc. Am. 105(5), 2590-2600 (1999).

11. S. Sinayoko, P. Joseph, and A. McAlpine, "Multimode radiation from an unflanged, semi-infinite circular duct with uniform flow,” J. Acoust. Soc. Am. 127(4), 2159-2168.

12. W. E. Zorumski, "Generalized radiation impedances and reflection coefficients of circular and annular ducts," J. Acoust. Soc. Am. 54(6), 1667-1673 (1973).

13. ${ }^{1}$ A. N. Norris and I. C. Sheng, "Acoustic radiation from a circular pipe with an infinite flange," J. Sound Vib. 135(1), 85-93 (1989).

14. J. C. Wendoloski, F. R. Fricke, and R. C. McPhedran, "Boundary-conditions of a flanged cylindrical pipe," J. Sound Vib. 162(1), 89-96 (1993).

15. Y. Nomura, I. Yamamura, and S. Inawashiro, "On the acoustic radiation from a flanged circular pipe,” J. Phys. Soc. Japan. 15(3), 510-517 (1960). 
16. H. J. Bom and T. J. Park, "A series solution for acoustic radiation from a flanged circular pipe," Acustica. 80, 315-316 (1994).

17. N. Amir, H. Matzner, and S. Shtrikman, "Acoustics of a flanged cylindrical pipe using singular basis functions,” J. Acoust. Soc. Am. 107(2), 714-724 (2000).

18. A. R. da Silva and G. P. Scavone, "Lattice boltzmann simulations of the acoustic radiation from waveguides," J. Phys. A. 40(3), 397-408 (2007).

19. A. R. da Silva, G. P. Scavone, and A. Lefebvre, "Sound reflection at the open end of axisymmetric ducts issuing a subsonic mean flow: A numerical study," J. Sound Vib. 327(3-5), 507-528 (2009).

20. M. A. Hamdi and J. M. Ville, "Sound radiation from ducts - theory and experiment," J. Sound Vib. 107(2), 231-242 (1986).

21. A. Selamet, Z. L. Ji, and R. A. Kach, "Wave reflections from duct terminations," J. Acoust. Soc. Am. 109(4), 1304-1311 (2001).

22. K. Gerdes, "The conjugated vs. The unconjugated infinite element method for the helmholtz equation in exterior domains," Comput. Methods Appl. Mech. 152(1-2), 125145 (1998).

23. D. S. Burnett, "A three-dimensional acoustic infinite element based on a prolate spheroidal multipole expansion,” J. Acoust. Soc. Am. 96(5), 2798-2816 (1994).

24. C. K. Lau and S. K. Tang, "Sound transmission across duct constrictions with and without tapered sections," J. Acoust. Soc. Am. 117(6), 3679-3685 (2005).

25. D. Givoli, "High-order local non-reflecting boundary conditions: A review," Wave Motion. 39(4), 319-326 (2004).

26. R. J. Astley, "FE mode-matching schemes for the exterior helmholtz problem and their relationship to the fe-dtn approach," Commun. Numer. Meth. Engng. 12(4), 257-267 (1996).

27. ASHRAE Handbook, HVAC Applications, (American Society of Heating, Refrigerating and Air-Conditioning Engineers, 2007) p. 47.20.

28. P.M. Morse and K.U. Ingard, Theoretical Acoustics, (McGraw Hill, New York, 1968) pp 332-338.

29. R. Kirby and J.B. Lawrie, "A point collocation approach to modelling large dissipative silencers," J. Sound Vib. 286, 313-339 (2005).

30. J.B. Lawrie and I.M.M. Guled, "On tuning a reactive silencer by varying the position of an internal membrane," Journal of the Acoustical Society of America 120 (2006) pp 780790. 
31. R. Kirby, "A comparison between analytic and numerical methods for modelling automotive dissipative silencers with mean flow," J. Sound Vib. 325(3), 565-582 (2009).

32. J.B. Lawrie and R. Kirby, "Mode matching without root finding: Application to a dissipative silencer," Journal of the Acoustical Society of America 119 (2006) pp 20502061.

33. CIBSE, Guide B5, Noise and Vibration Control for HVAC, (Chartered Institution of Building Services Engineers, UK, 2005) p. 5-11.

34. European Standard EN ISO 1505, "Ventilation for buildings - Sheet metal air ducts and fittings with rectangular cross-section- Dimensions," (1998) 
Table I. The influence of the location of $\Gamma_{A}$ and $\Gamma_{B}$ on solution convergence for a flanged circular duct.

\begin{tabular}{ccc|ccc}
\hline \multirow{2}{*}{$L / a$} & \multicolumn{2}{c|}{$\delta / a$} & & \multicolumn{2}{c}{$\delta / a$} \\
\cline { 2 - 3 } \cline { 5 - 6 } & $k a=0.1$ & $k a=1$ & & $k a=0.1$ & $k a=1$ \\
\hline 0 & 0.818723 & 0.606051 & 1 & 0.820389 & 0.607369 \\
0.04 & 0.816917 & 0.604392 & 2 & 0.816849 & 0.604328 \\
0.08 & 0.816852 & 0.604332 & 3 & 0.816849 & 0.604328 \\
0.2 & 0.816849 & 0.604328 & 4 & 0.816849 & 0.604328 \\
0.6 & 0.816849 & 0.604328 & 5 & 0.816849 & 0.604328 \\
1.0 & 0.816849 & 0.604328 & 6 & 0.816849 & 0.604328 \\
\hline
\end{tabular}


Table II. The influence of the location of $\Gamma_{\mathrm{B}}$ on solution convergence for an unflanged circular duct.

\begin{tabular}{ccc}
\hline \multirow{2}{*}{$\mathrm{R} / \mathrm{a}$} & \multicolumn{2}{c}{$\delta / \mathrm{a}$} \\
\cline { 2 - 3 } & $\mathrm{ka}=0.1$ & $\mathrm{ka}=1$ \\
\hline 1.5 & 0.617629 & 0.516580 \\
2 & 0.611119 & 0.524223 \\
3 & 0.608399 & 0.524509 \\
4 & 0.607773 & 0.523434 \\
5 & 0.607556 & 0.523882 \\
6 & 0.607461 & 0.523947 \\
\hline
\end{tabular}




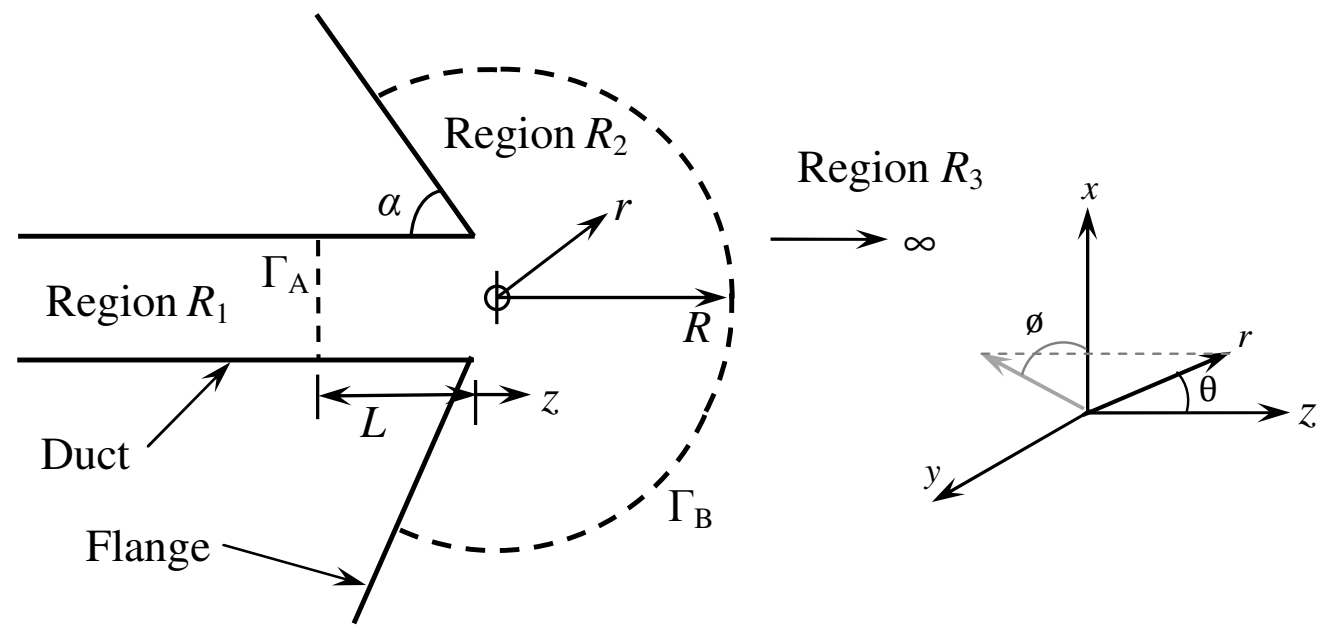

Fig. 1. Geometry of duct. 
Duan, JASA

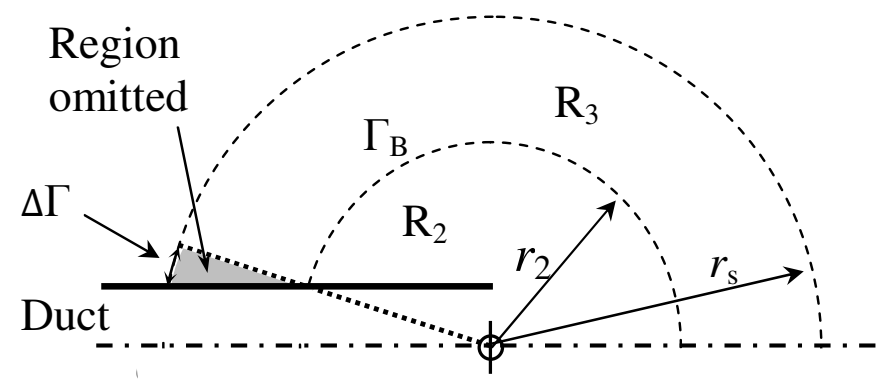

Fig. 2. The model envelope for an unflanged pipe. 


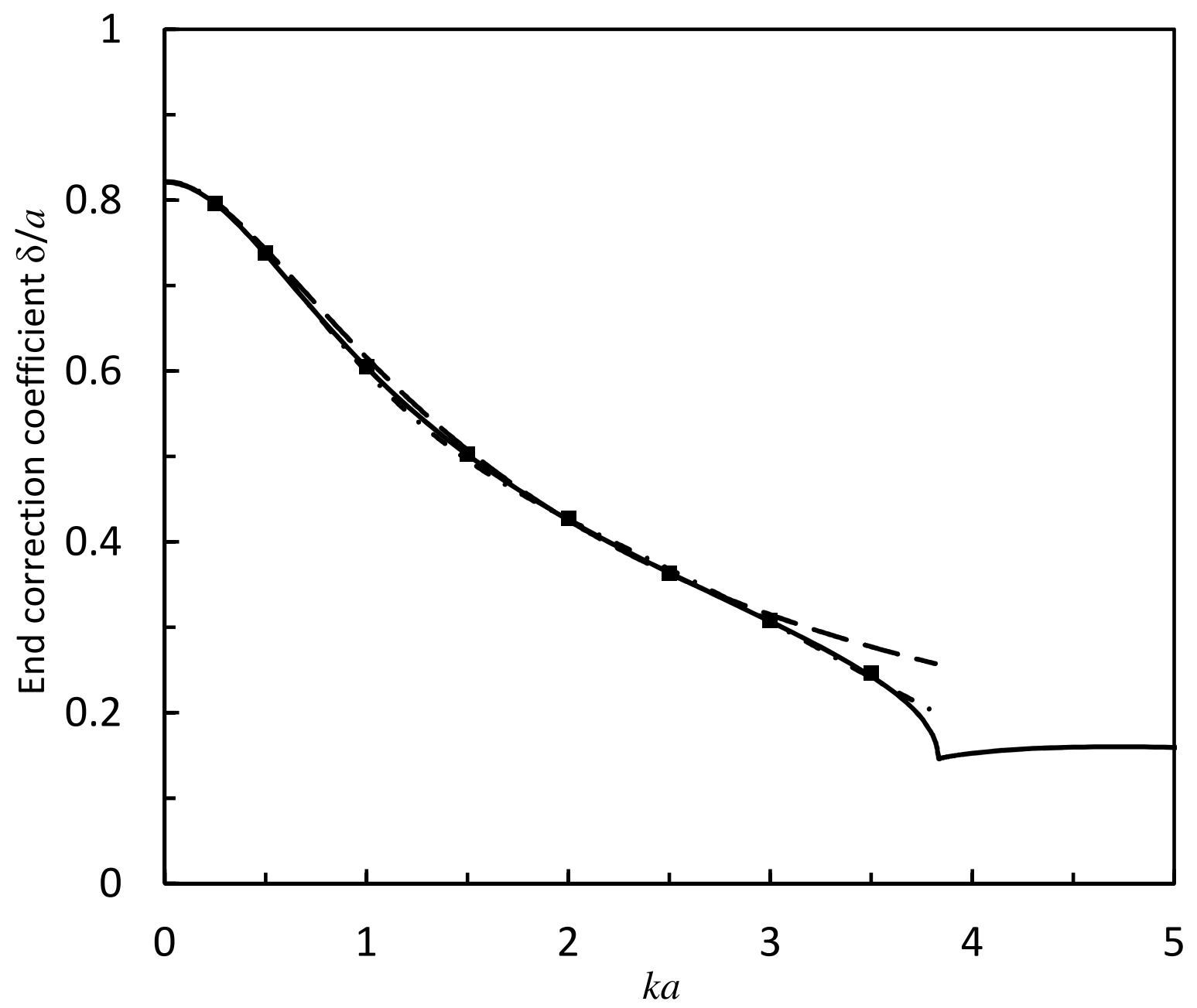

Fig. 3. End correction coefficient for flanged circular duct: - — FE predictions; $\mathbf{\square}$, Nomura et al. ${ }^{15} ;-\cdot-\cdot-$, Silva et al. ${ }^{6} ;---$, Norris and Sheng ${ }^{13}$. 


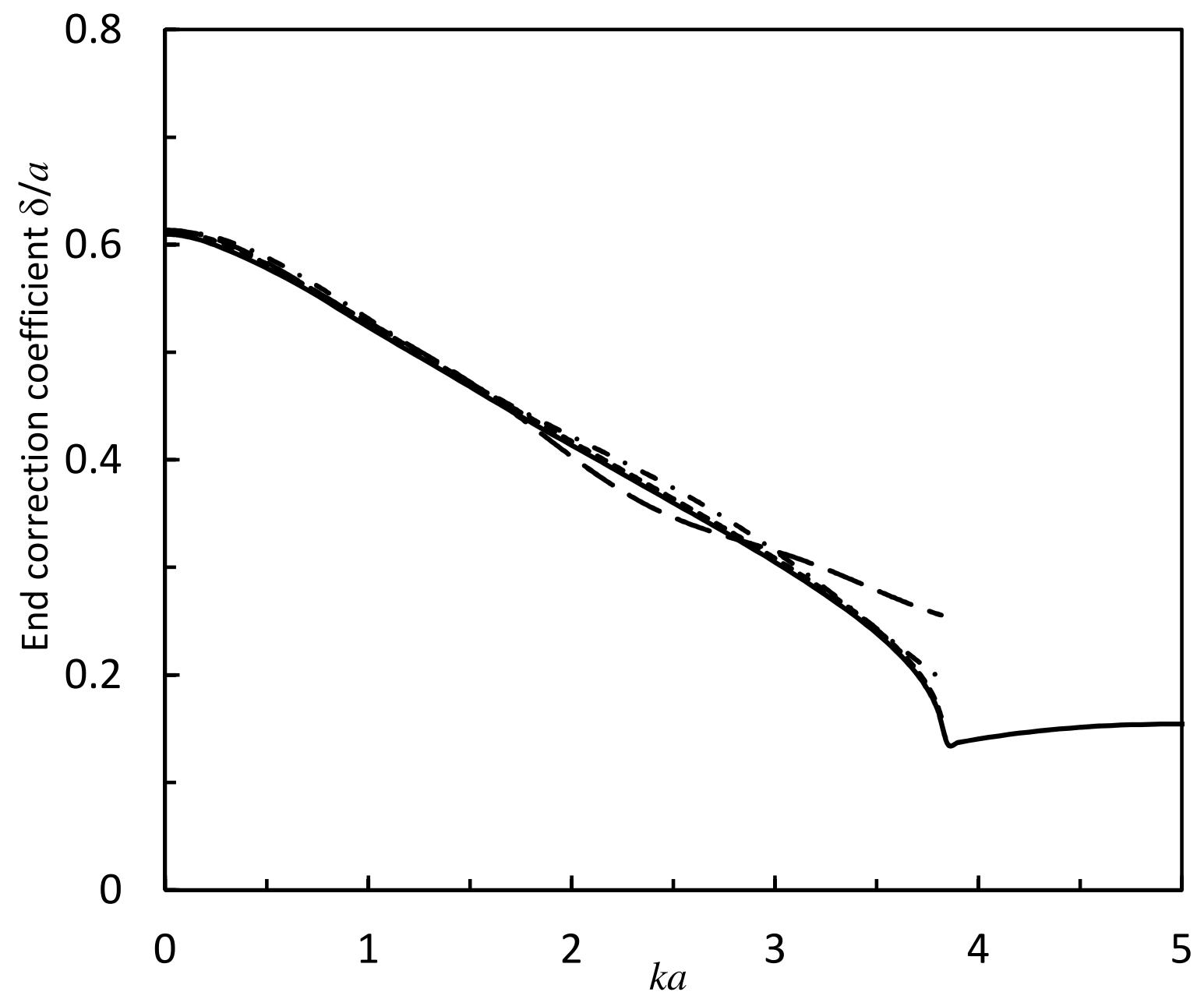

Fig. 4. End correction coefficient for unflanged circular duct: ——, FE predictions; - - - - -, Levine and Schwinger ${ }^{3}$ (overlays FE);- - - - Silva et al. ${ }^{6} ;---$, Dalmont et al..$^{5}$ 


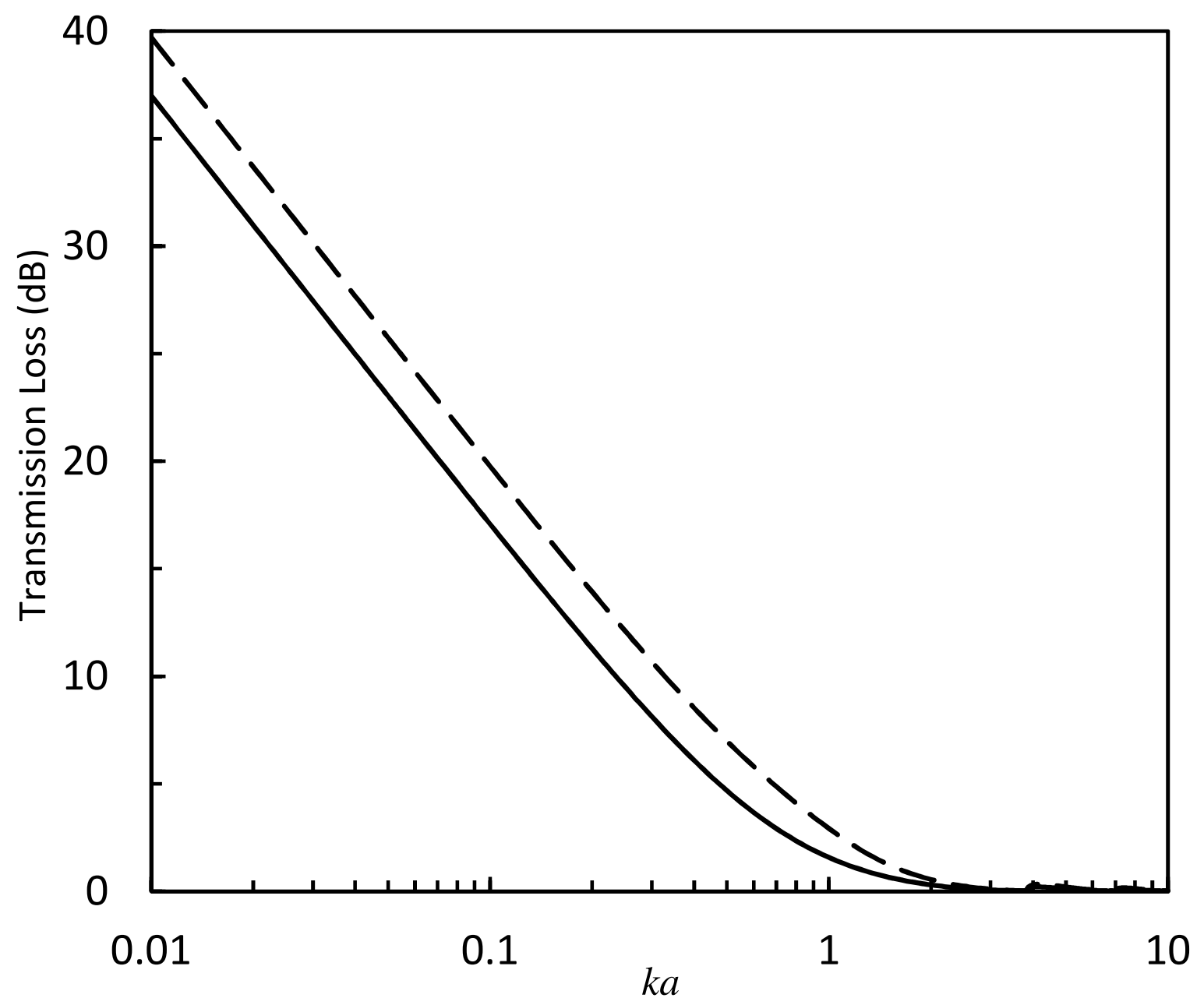

Fig. 5. FE predictions of TL for circular duct: ——, flanged; - - - unflanged. 


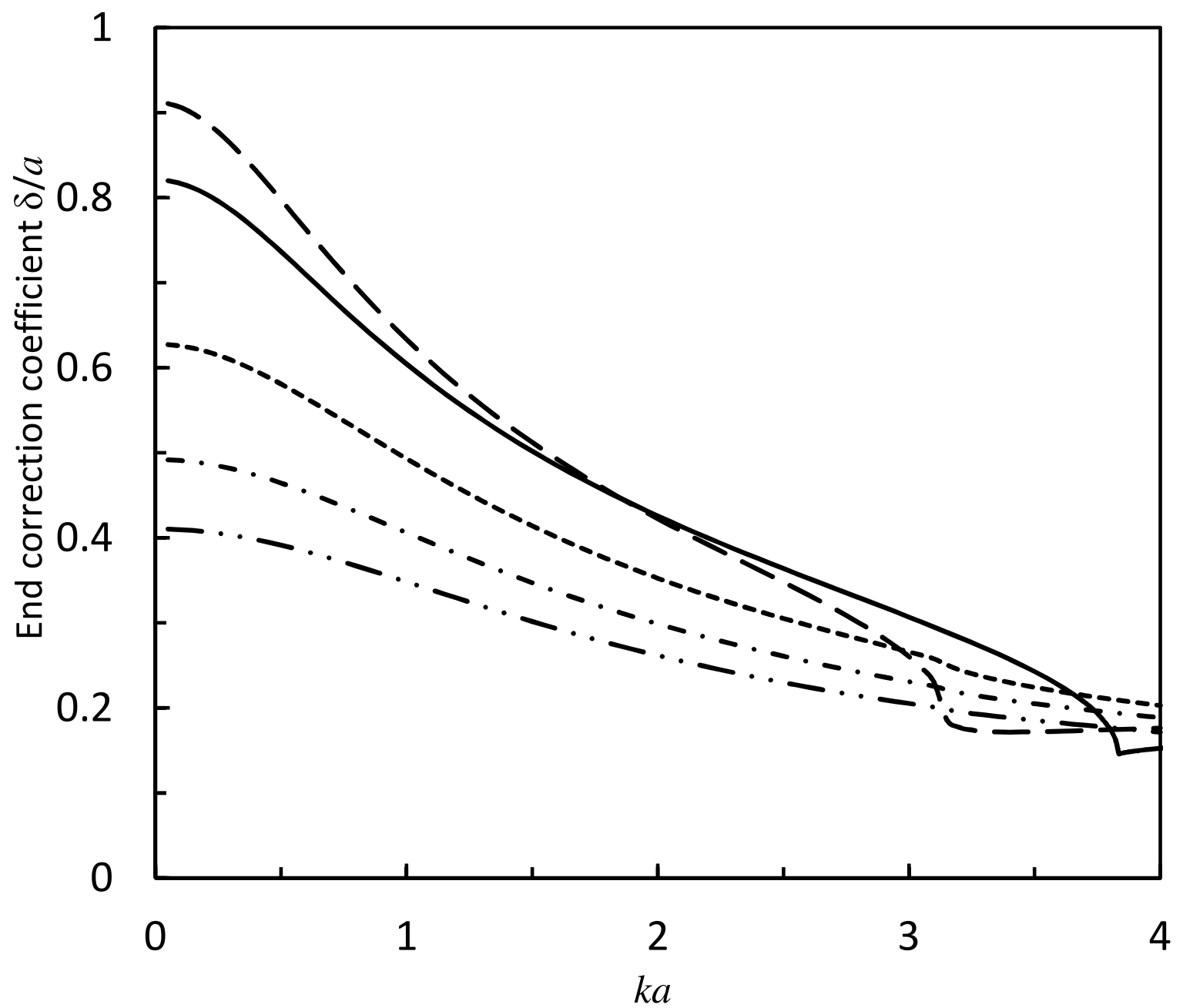

Fig. 6. FE predictions of end correction coefficient for an unflanged rectangular duct: ——, circular duct; - - , $\quad ;-\cdots, \quad ;-\cdots-\cdots, \quad ;-\cdots-\cdots-$, 


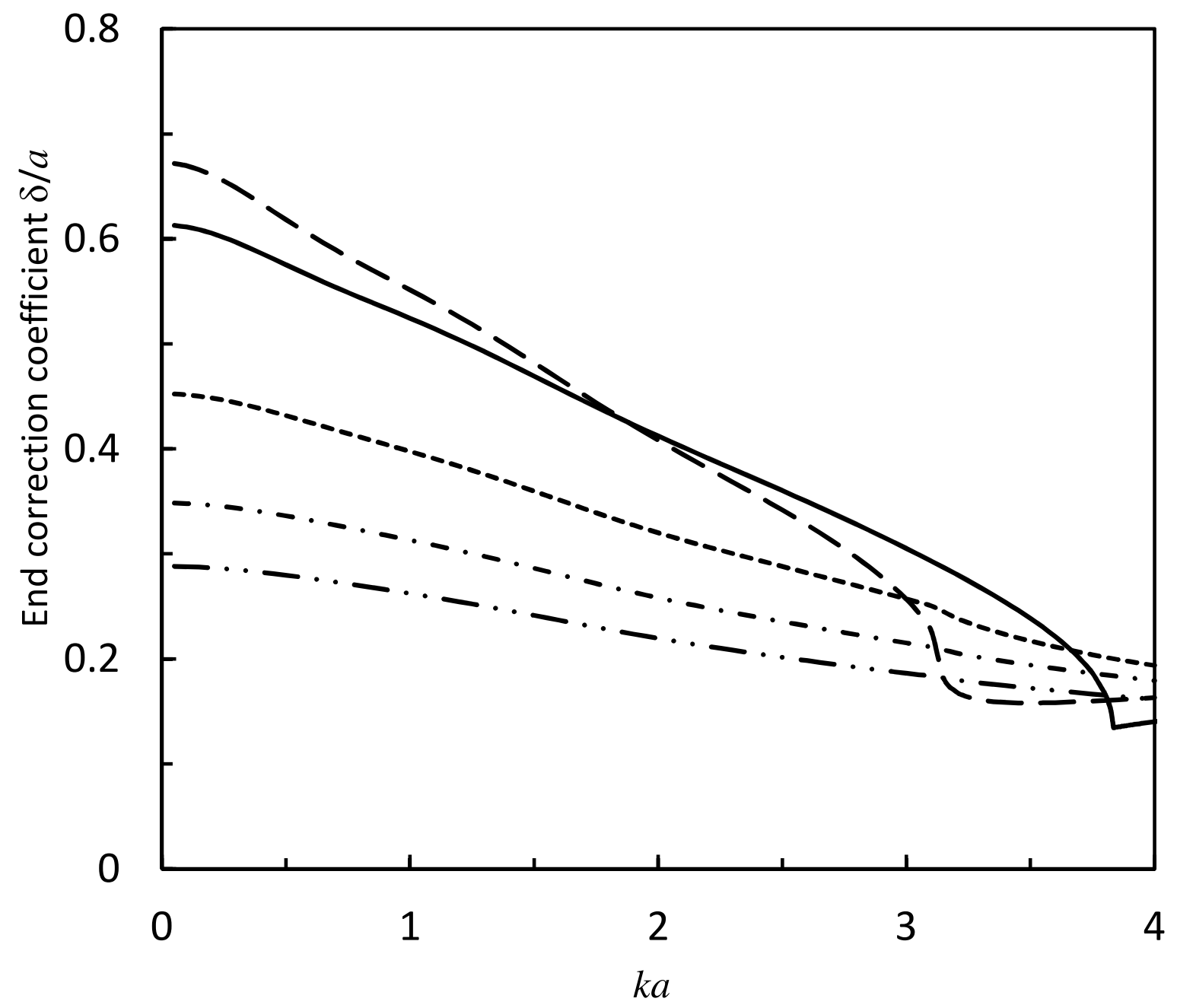

Fig. 7. FE predictions of end correction coefficient for a flanged rectangular duct: circular duct; - - , $\quad ;---, \quad ;-\cdots-\cdots-, \quad ;-\cdots-\cdots-$, 


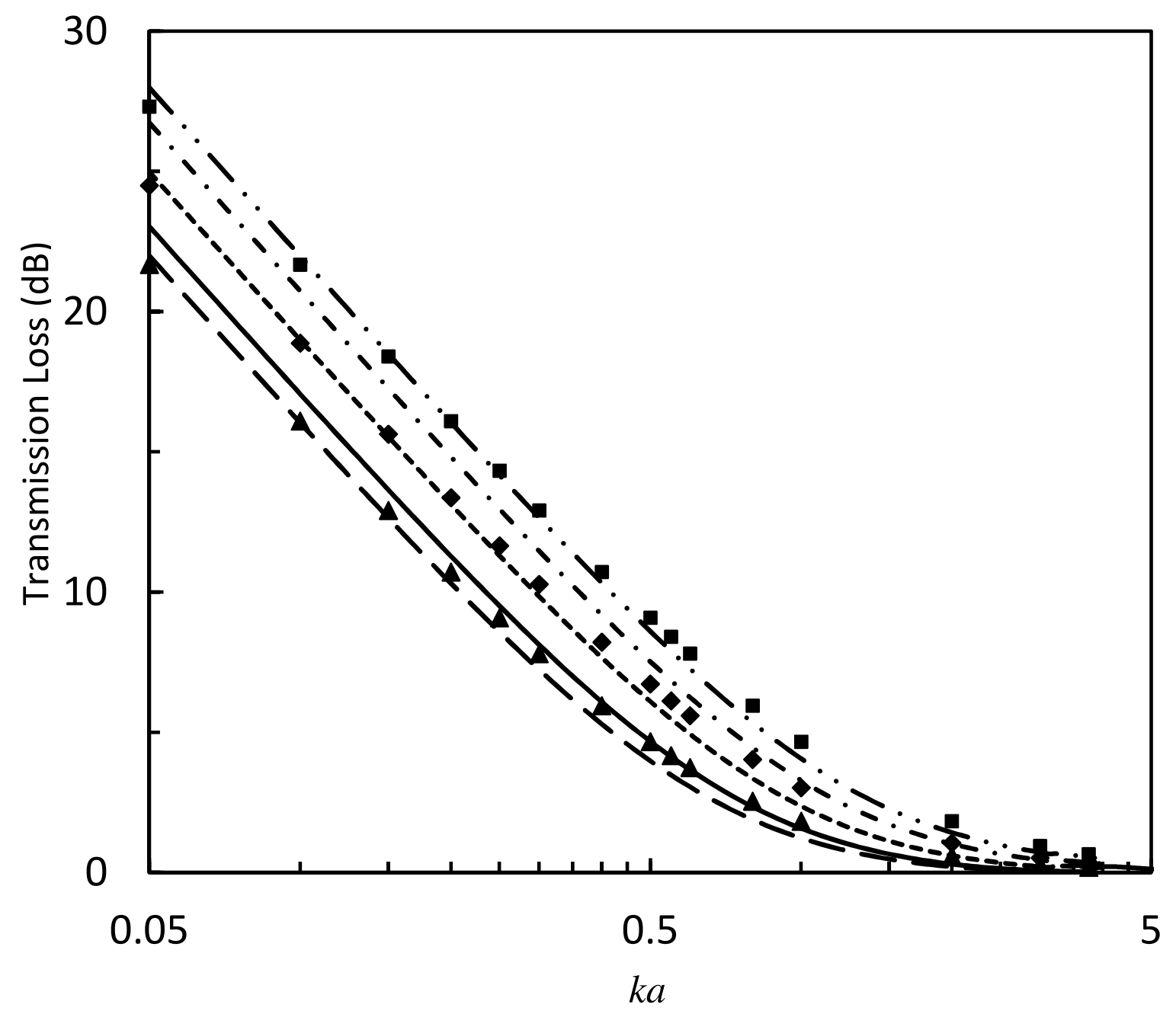

Fig. 8. Transmission loss predictions for flanged rectangular duct: - , circular duct; - -
$-$
; - - - -
$;-\cdot-\cdot-$

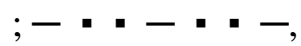
$\boldsymbol{\Lambda}$, CIBSE, ;
$\checkmark$, CIBSE, $\quad ; \mathbf{\square}$, CIBSE, 